\title{
Correction to: Scheduling Algorithms for Reconfigurable Systems
}

\section{Correction to:}

Chapter 3 in: K. Guha et al., Self Aware Security for Real Time Task Schedules in Reconfigurable Hardware Platforms, https://doi.org/10.1007/978-3-030-79701-0_3

In the original version of the book, the following belated corrections have been incorporated: In Ref. [MVGR13] of Chapter 3, the first author's name has been corrected from "A. Medina Villanueva" to "A. Morales-Villanueva" and the second author's name from "A.G. Ross" to "A. Gordon-Ross". The book and the chapter have been updated with the changes. 